\title{
Idiopathic Thyroid Abscess - A Rare Occurrence
}

\author{
Mansharan Kaur Chainchel Singh ${ }^{1,2}$ and Anushya Vijayanathan ${ }^{3}$ \\ 1. Faculty of Medicine, Universiti Teknologi MARA Sungai Buloh, Selangor, Malaysia; 2. Institute of Pathology, Laboratory and Forensic \\ Medicine (I-PPerForM), Universiti Teknologi MARA, Sungai Buloh, Selangor, Malaysia 3. Department of Biomedical Imaging, Faculty of \\ Medicine, University of Malaya, Kuala Lumpur, Malaysia.
}

DOI: https://doi.org/10.17925/EE.2019.15.1.42

$\mathrm{T}$ hyroid abscesses are rare as the gland has a rich blood supply, well-developed capsule and is high in iodine. However, clinicians must be aware of this fact and make an early diagnosis as it can lead to serious morbidity if left untreated. Infection may involve one or both lobes of the thyroid gland, although the left lobe is more often affected and is successfully treated with surgery and antibiotics. This case study involves a 22-year-old woman with a history of painful swelling over the neck accompanied by low-grade fever without any underlying cause. Both the ultrasound and computed tomography revealed a heterogeneous mass within the left lobe of the thyroid gland. Fine needle aspiration revealed an abscess collection and culture Staphylococcus aureus had grown. She was successfully treated with intravenous and oral amoxicillin clavulanate and no surgical treatment was carried out. In conclusion, thyroid abscesses are rare as the gland is resistant to infection and must be differentiated from an infected branchial cleft cyst or sinus, infected thyroid tumour or lymphadenitis in the region of the thyroid in order to avoid serious morbidity if left untreated.

\section{Keywords}

Fever, pain, swelling over the neck, thyroid abscess

Disclosures: Mansharan Kaur Chainche Singh and Anushya Vijayanathan have no relevant conflicts of interest to declare.

Review Process: Double-blind peer review.

Compliance with Ethics: All procedures were followed in accordance with the responsible committee on human experimentation and with the Helsinki Declaration of

1975 and subsequent revisions, and informed consent was received from the patient involved in this case study.

Authorship: The named authors meet the criteria of the International Committee of Medical Journal Editors for authorship for this manuscript, take responsibility for the integrity of the work as a whole and have given final approval for the version to be published.

Received: 31 July 2018

Accepted: 10 October 2018

Citation: European Endocrinology. 2019;15(1):42-3

Corresponding Author: Mansharan Kaur

Chainchel Singh, Institute of Pathology, Laboratory

and Forensic Medicine (I-PPerForM), Level 4

Academic Building Universiti Teknologi MARA,

Sungai Buloh, Jalan Hospital, 47000 Selangor,

Malaysia. E: mansharan@salam.uitm.edu.my

Support: No funding was received in

the publication of this article.
Thyroid abscess is rare, accounting for less than $0.7 \%$ of surgical pathologies involving the thyroid gland. ${ }^{1,2}$ This is attributed to its rich blood supply and lymphatic drainage, high concentration of iodine that inhibits bacterial growth, protective fibrous capsule, and fascial planes separating it from other neck structures. ${ }^{2-4}$ Patients with a thyroid abscess usually associate their fever with a painful swelling in the anterior aspect of the neck and the diagnosis is made from the physical finding of a neck swelling, diagnostic imaging, fine needle aspiration and laboratory examination. ${ }^{5}$ The diagnosis and management of thyroid abscesses must be performed quickly in order to avoid complications such as septicaemia, paralysis of the vocal cords, retropharyngeal abscess and suppurative mediastinitis occasionally leading to osteomyelitis or septic thrombophlebitis. ${ }^{2}$

\section{Case report}

A 22-year-old Indonesian female who had just given birth to her first child 4 months previously came to the medical clinic with a history of swelling over the neck associated with pain and lowgrade fever. For the previous month the fever seemed more prominent at night with a sudden increase in size of the swelling and difficulty in swallowing for the previous week. There was no evidence of hand tremors or hoarseness of voice, which would suggest thyroid dysfunction. Nor was there a history of contact with tuberculosis to suggest tuberculosis of the thyroid gland. The physical examination revealed a painful swelling measuring $3 \times 3 \mathrm{~cm}$ in size over the left side of the neck, which moved on deglutition.

The patient was subjected to a series of radiological examinations, which included an ultrasound of the neck, a radionuclide study of the thyroid gland and a computed tomography (CT) scan of the neck. The ultrasound revealed a heterogeneous mass just below the subcutaneous tissue involving the left lobe of thyroid measuring $2.3 \times 2.5 \mathrm{~cm}$ in size. The mass showed increased internal vascularity; however, as the mass was not arising from a vessel, the diagnosis of abscess was made (Figure 1). CT scan of the neck revealed an inhomogeneously enhancing mass measuring $3.0 \times 3.0 \times 4.0 \mathrm{~cm}$ in size with cystic areas involving the left lobe of the thyroid. The adjacent neck vessels and the right lobe of the thyroid gland were normal (Figure 2).

Blood investigations revealed that thyroxine (T4) levels were raised at $28.6 \mathrm{pmol} /$ litre (normal range 10-23 pmol), thyroid stimulating hormone (TSH) was low at $0.1 \mathrm{mIU} /$ litre (normal range $0.5-5 \mathrm{mIU} / \mathrm{l}$ ) and erythrocyte sedimentation rate (ESR) was raised at $>140 \mathrm{~mm}$ (normal range $<30 \mathrm{~mm}$ ). The chest $\mathrm{x}$-ray was normal and the Mantoux test was negative. A fine needle aspiration of the mass showed a focus of necrosis and a small pocket of abscess lined by granulation tissue. There was no evidence of malignant cells to suggest malignancy and no evidence of lymphocytic infiltration to suggest thyroiditis. Culture of the pus from the abscess obtained during the fine needle aspiration grew Staphylococcus aureus. 
Figure 1: Ultrasound showing a heterogenous mass of the left thyroid lobe (arrows)

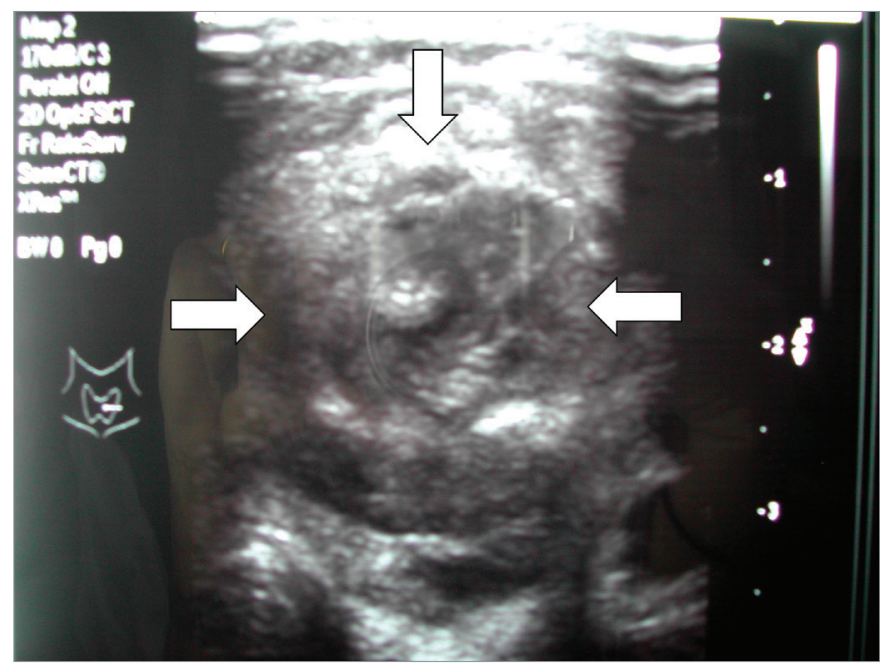

Figure 2: Axial computed tomography scan of the neck showing diffuse enlargement and heterogenous enhancement of the left thyroid lobe and isthmus (arrows) with a low attenuation area within it (arrows)

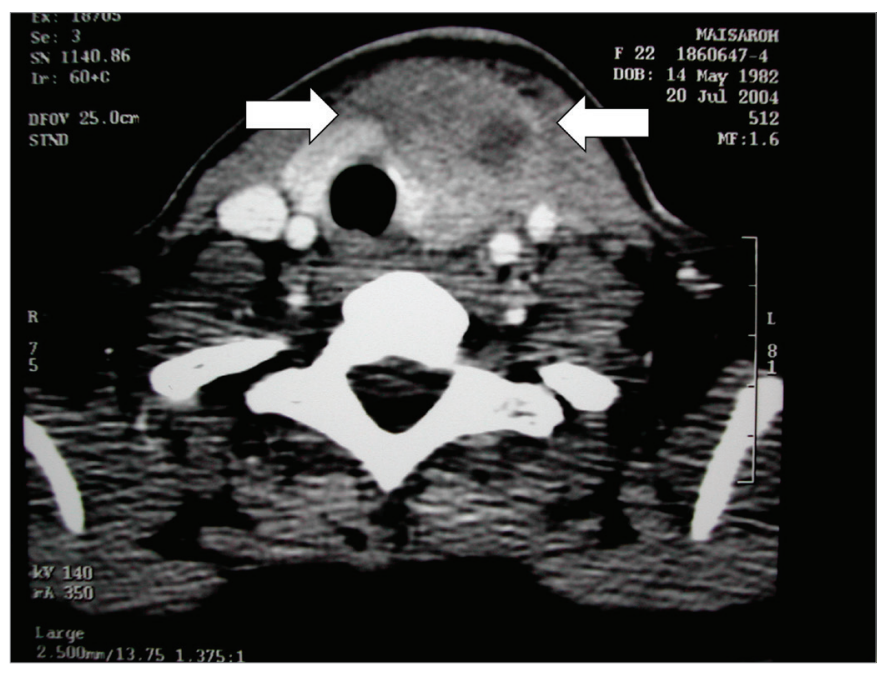

The patient was then started on intravenous amoxicillin clavulanate $600 \mathrm{mg} 8$ hourly for 5 days. She responded to antibiotic treatment and did not require any surgical drainage as her fever and pain settled. She was later switched to oral amoxicillin clavulanate $500 \mathrm{mg} 8$ hourly for 7 days. Repeat blood investigations done for ESR, TSH and T4 hormone levels showed normal values with an ESR of $30 \mathrm{~mm}$, TSH of $2.5 \mathrm{mIU} / \mathrm{l}$ and a T4 level of $20 \mathrm{pmol} / \mathrm{l}$.

\section{Discussion}

Thyroid abscesses are rare as the gland is resistant to infection. The patient usually presents with fever and a painful neck swelling, which would give rise to a number of differential diagnoses like thyroiditis, fungal or tuberculous infection of the thyroid gland and thyroid abscess.
Other causes to be considered would be an infected branchial cleft cyst or sinus, infected thyroid tumour and lymphadenitis in the region of the thyroid. Differentiating these entities may be difficult clinically, thus the use of imaging and needle aspiration for cytology and culture is important. ${ }^{1}$ Recurrent thyroid abscess, especially in children, is mostly due to a pyriform sinus fistula and persistence of a thyroglossal duct remnant. ${ }^{3}$ In adults, long-standing thyroid goiter, thyroid malignancy and immunocompromised states are known predisposing factors. This case was unusual because we were not able to identify an underlying thyroid pathology or anatomic anomaly.

The exact pathogenesis of thyroid abscess is unclear except in cases of direct trauma; other possible routes of infection would include haematogenous spread (via the superior thyroid, inferior thyroid and thyroidea ima arteries), lymphogenous spread, internal fistula from a pyriform sinus tract and via a patent thyroglossal duct cyst. The most common organisms cultured from thyroid abscess cavities include Group A haemolytic Streptococcus, Staphylococcus and Pneumococcus. ${ }^{1-4}$

Studies have shown that thyroid abscess is more common in women compared with men, though some studies report an equal incidence. ${ }^{3,4}$ Thyroid abscesses also more commonly involve the left lobe compared with the right lobe as observed in this case report. A diagnosis of thyroid abscesses is important and clinicians need to make an early diagnosis to avoid serious morbidity if left untreated. Blood investigations, such as raised total white blood cell count and ESR, indicate ongoing inflammatory processes whereby infection, e.g. abscess, is one of the most common causes. Ultrasound often demonstrates a heterogeneous echotexture of the thyroid gland with a superimposed anechoic or hypoechoic mass, while CT findings of abscesses vary depending on the stage of infection with heterogeneous enhancement of the thyroid.

Histological examination of a thyroid abscess usually shows haemorrhage, focal necrosis and suppurative small miliary abscess, or a purulent pocket involving the entire lobe with a cavity lined by granulation tissue and surrounding zone of fibroplasias. ${ }^{2.6}$ This was similar to our case, which showed a focus of necrosis and a small pocket of abscess lined by granulation tissue.

Management of thyroid abscess requires drainage either percutaneously or by incision via a collar incision and simultaneous antibiotic treatment. This treatment is usually with cephalosporins to avoid complications such as septicaemia, local spread via fascial planes, retropharyngeal abscess, rupture of abscess into the trachea or esophagus and internal jugular vein thrombophlebitis. ${ }^{2}$ There have, however, been instances where patients have been successfully treated with antibiotic treatment alone not requiring any surgical drainage as in this case. ${ }^{7.8}$

\section{Conclusion}

In conclusion, thyroid abscesses are rare as the gland is resistant to infection and they must be differentiated from an infected branchial cleft cyst or sinus, infected thyroid tumour or lymphadenitis in the region of the thyroid. A diagnosis of thyroid abscesses is important and clinicians need to make an early diagnosis to avoid serious morbidity if left untreated. $\square$

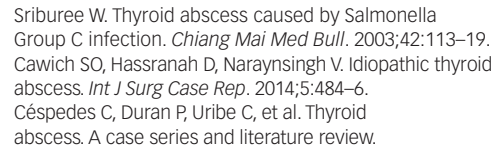

Endocrinol Nutr. 2013;60:190-6.

Nirhale D, Athavale V, Goenka G, Bhatia M. Thyroid abscess: A rare entity? Thyroid Res Pract. 2014;11:131-2.

Astl J, Kuchynková Z, Taudy M, Betka J Thyroid abscess at an adolescent age Int $J$ Pediatr Otorhinolaryngol. 2003:67:1375-8. De Souza RE, Dilip A. Thyroid abscess with cutaneous fistula:
Case report and review of the literature. Thyroid Science. 2008:3:1-4.

Shah SS, Baum SG. Diagnosis and management of infectious thyroiditis. Curr Infect Dis Rep. 2000:2:147-53. Sarfaraz M, Hasan SR Qureshi Z Thyroid abscess in children and the world gets stranger. J Rare Disord Diagn Ther. 2018:4:1-3. 